\title{
Nifedipine in Scleroderma Ulcerations
}

\author{
Thomas Y. Woo, M.D., Reynold C. Wong, M.D., Janis P. Campbell, M.D., \\ Michael T. Goldfarb, M.D., John J. Voorhees, M.D., \\ and JefFrey P. Callen, M.D.
}

From the Departments of Dermatology,

University of Michigan Medical School,

Ann Arbor, Michigan and

University of Louisville School of Medicine,

Louisville, Kentucky

ABSTRACT: Cutaneous ulcerations may be due to a variety of causes, including vasculitis, infections, arterial insufficiency, and microvascular damage. The net effect is diminished blood flow to the skin. Nifedipine, a calcium antagonist, has been shown to improve cutaneous blood flow and to alleviate reactive vasospastic ischemia (Raynaud's phenomenon). The authors report an ischemic ulcer of scleroderma showing visible improvement with nifedipine therapy.

Progressive systemic scleroderma (PSS) is a debilitating disorder with a multitude of clinical manifestations, including bound-down skin, Raynaud's phenomenon, esophageal dysfunction, pulmonary fibrosis, renal failure, and cutaneous ulcerations secondary to vascular insufficiency. The cause of increased collagen deposition in body tissues in scleroderma remains an enigma. Recent research has implicated circulating platelet aggregates and elevated beta-thromboglobulin ${ }^{1}$ as well as antibodies directed against the endothelial cell. ${ }^{2}$ This results in small vessel damage leading to increased deposition of collagen by fibroblasts.

Raynaud's phenomenon is an arteriolar vasospastic condition that is often seen in PSS. A variety of medications have been used to treat Raynaud's phenomenon. These include oral agents, such as phenoxybenzamine, alpha-methyldopa, guanethidine, captopril, and verapamil. Also, intraarterial reserpine, intravenous prostacyclin, topical nitroglycerin, sympathectomy and

Address for reprints: Thomas Y. Woo, M.D., 142320 Street, N.W., Calgary, Alberta, Canada T2N 2 K7. biofeedback have been used. ${ }^{3}$ Recently, Kahan and his associates ${ }^{4}$ reported that the use of oral nifedipine (a calcium channel blocker) in patients with Raynaud's phenomenon resulted in a decrease in the mean number of digital vasospastic attacks from 29.5 to 4.3 per week concurrent with a subjective improvement in 14 of 16 patients. They also found that nifedipine was effective in a single-blinded crossover study of four patients. Raynaud's phenomenon, which involves transient cutaneous ischemia due to a vasospastic condition, is somewhat similar to the cutaneous ischemia from microvascular disease, which occurs in ulcers of scleroderma. Therefore, this report prompted us to use nifedipine in treating a cutaneous ulcer that was unresponsive to conservative therapy in a patient with PSS.

\section{Case Report}

A 68-year-old white woman was seen in consultation at the Department of Dermatology, University of Michigan with a 3-year history of an ulcer on the medial aspect of her left foot. The etiology of the ulcer was uncertain. She had a 15-year history of PSS with Raynaud's phenomenon, dysphagia, and cutaneous sclerosis. Two years prior to her presentation, an angiography of the left leg had revealed an arterial blockage. In an effort to increase blood supply to the foot, she subsequently had a therapeutic left femoral popliteal bypass in January 1981 and a left lumbar sympathectomy in October 1981, but the ulcer persisted. She was first seen at the University of Michigan in February 1982, and extensive inpatient and outpatient treatment with bedrest, whirlpool, topical benzoyl peroxide, and systemic antibiotics were tried with little success over a period of 8 months.

She was in no acute distress. Physical findings included a 3 $\times 3-\mathrm{cm}$, purulent, shallow ulcer with discrete margins located superior and anterior to the left medial malleolus (Fig. 1). Although the left leg had a femoral pulse, the popliteal, posterior tibial, and dorsalis pedis pulses were undetectable. The left foot was also cold to touch. The skin of the hands, feet, and ankles was very taut. There were periungual telangiectasias on the fingers and sclerodactyly. She had a beaked nose and radial furrowing around the mouth with bound-down facial 


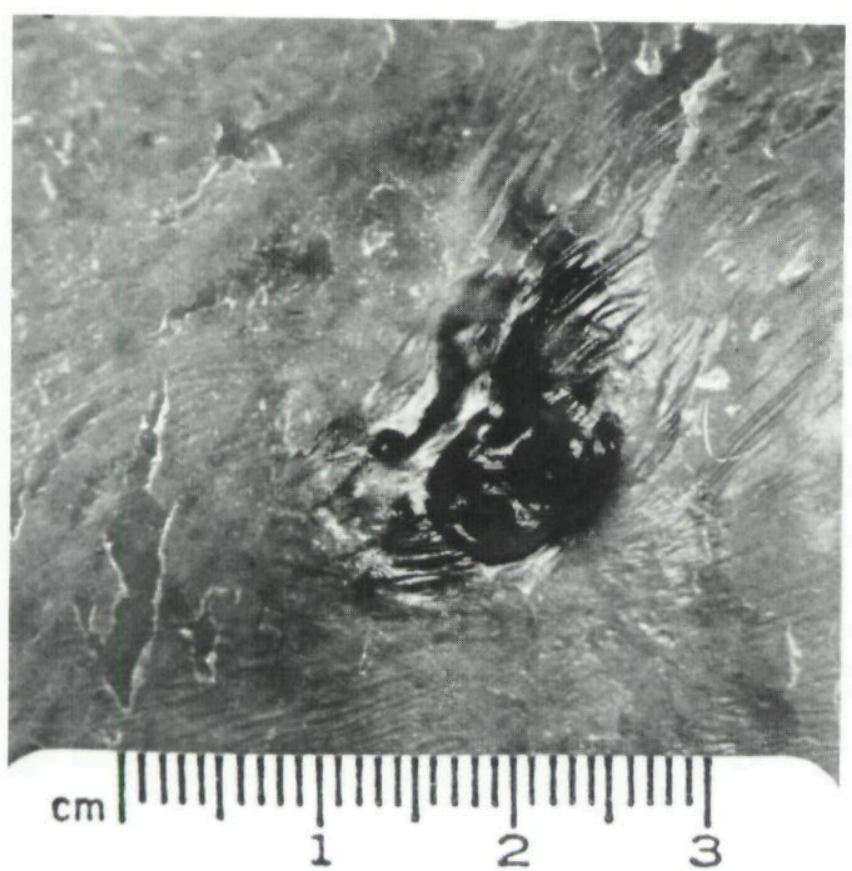

FiG. 1. Appearance of the ulcer at the end of one month therapy with nifedipine. Note outline of previous ulceration.

skin. Similarly, the rest of her skin was sclerotic and bounddown.

Pertinent laboratory findings revealed an elevated Westergren erythrocyte sedimentation rate of 100 . Systemic manifestations of PSS included findings consistent with moderate pulmonary fibrosis as demonstrated by a reduced functional vital capacity of $1.95 \mathrm{~L}$, forced expiratory volume at 1 second of $1.34 \mathrm{~L}$, and an abnormal carbon monoxide diffusion of 8.72 . The upper gastrointestinal series revealed decreased esophageal motility of the distal esophagus consistent with PSS. An x-ray of the left ankle failed to reveal any osteomyelitis. Cultures of the ulcer grew Pseudomonas aeruginosa (sensitive to sulfamethoxazole-trimethoprim) and coagulase positive staphylococcus (sensitive to erythromycin). Antinuclear, anticentromere, Scl-70 (scleroderma antibody) and anti-nDNA antibodies were negative. Chest $\mathrm{x}$-ray was within normal limits. The rest of the laboratory evaluation was unremarkable.

Local treatment to the ulcer was instituted and consisted of daily whirlpool and Op-Site dressings. She also received a 10-day course of erythromycin base $(500 \mathrm{mg}$ four times daily) and sulfamethoxazole $400 \mathrm{mg}$ /trimethoprim $80 \mathrm{mg}$ (two tablets four times daily). No evidence of healing occurred during the course of antibiotic therapy despite adequate wound care. The patient was then given a trial of oral nifedipine at $10 \mathrm{mg}$ three times daily. This was slowly increased over a period of 7 days to $40 \mathrm{mg}$ three times daily. Her only side effect was dizziness (due to slight postural hypotension). Within 2 weeks, the foot became pinker in color and warm to touch. Over the course of 1 month, the ulcer became filled with granulation tissue and had totally reepithelialized approximately threequarters of its surface (Fig. 1). At this time, she was discharged on nifedipine $10 \mathrm{mg}$ three times daily (to avoid orthostatic hypotension), Op-Site, and home whirlpool. Follow up 2 months later revealed continual improvement with only a $2-\mathrm{mm}$ area of healing tissue (Fig. 2). The slower healing was

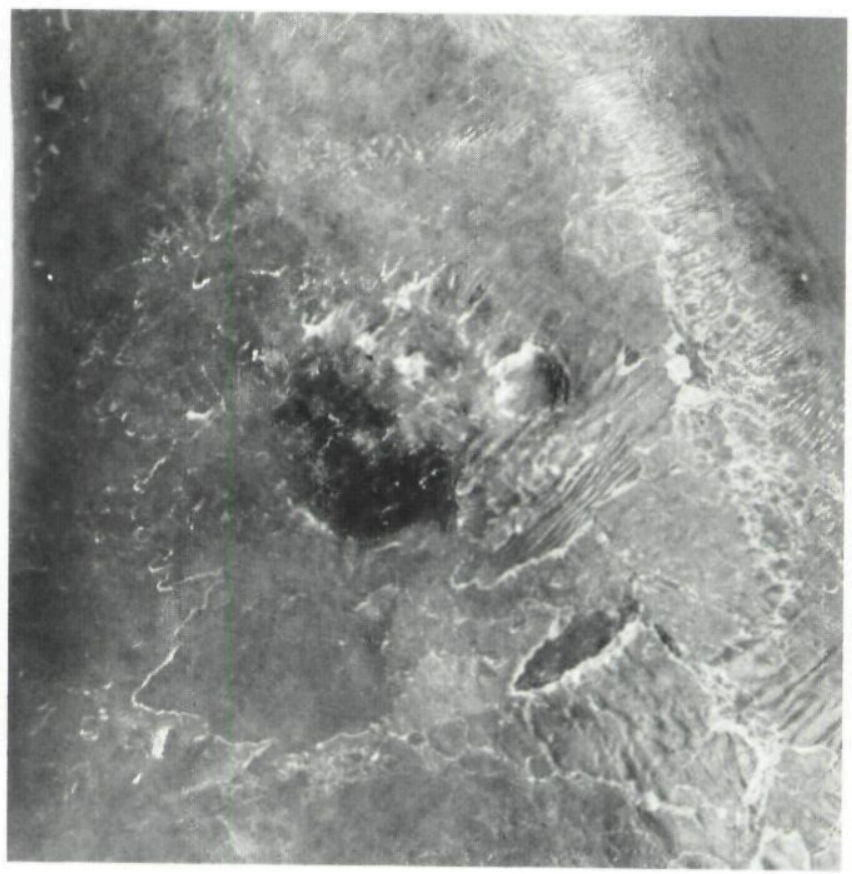

FIG. 2. Appearance of the ulceration after three months of nifedipine. Note the total reepithelialization of the ulcer.

possibly due to the reduced nifedipine dosage. There was no improvement in the PSS itself.

\section{Comment}

The dramatic resolution of the ulceration of 3 years duration in this patient suggests that nifedipine may be a useful therapeutic agent in the therapy of ulcers in patients with scleroderma. Refractory digital and elbow ulcerations in a patient with severe Raynaud's phenomenon and PSS have been reported to respond to nifedipine. ${ }^{5}$ Nifedipine is a calcium channel blocker that functions by impairing transfer of calcium into the cell, resulting in a lower intracellular concentration of calcium. ${ }^{6}$ The net effect is a relaxation in the smooth muscle beds of the body. The vascular smooth muscles of the arteriolar bed are far more sensitive to the action of nifedipine that those associated with the venous bed. The net effect is increased arteriolar pressure with a concurrent increase in capillary pressure and an enhanced cutaneous blood flow. This improvement in cutaneous circulation following use of nifedipine has been documented both in vitro ${ }^{7}$ and in vivo. ${ }^{8}$ Of all the calcium channel blockers now commercially available for patient use, nifedipine is the most potent cutaneous vasodilator.

Nifedipine is rapidly absorbed when taken orally and reaches its peak serum concentration in 20-45 minutes, with a half-life of $2.5-3$ hours. The intact drug is $90 \%$ bound to plasma proteins, whereas the free acid metabo- 
lite is only $54 \%$ bound. Over $90 \%$ of the orally administered dose of nifedipine is excreted by the kidney in the first 24 hours. ${ }^{9}$ Adverse side effects are uncommon, but include palpitations, orthostatic hypotension, reflex tachycardia, dizziness, a nonspecific rash (erythematous pedal edema), and flushing. ${ }^{10}$

Our patient had PSS with a persistant ankle ulcer for many years. The etiology of the ulcer in this patient is unclear. It may have been due to microvascular disease, as seen in scleroderma, or to arterial occlusion. Attempts to treat the ulcer with conventional inpatient and outpatient therapy were unsuccessful; however, nifedipine in doses of $120 \mathrm{mg}$ daily proved efficacious. The optimal dosage for ischemic sclerodermatous ulcerations is unknown at this time. Reports indicate that nifedipine in doses of $30-120 \mathrm{mg}$ daily is effective for Raynaud's phenomenon. ${ }^{11-13}$

\section{Drug Names}

nifedipine: Procardia

sulfamethoxazole/trimethoprim: Bactrim, Septra

\section{References}

1. Kahaleh MB, Osborn I, Leroy EL: Elevated levels of circulating platelet aggregates and beta-thromboglobulin in scleroderma. Ann Intern Med 96:610, 1982
2. Cohen S, Johnson AR, Hurd E: Cytotoxicity of sera from patients with scleroderma. Arthritis Rheum 26:170, 1983

3. Peterson LL, Vorheis C: Raynaud's syndrome: Treatment with sublingual administration of nitroglycerin, swinging arm maneuver and biofeedback training. Arch Dermatol 119:396, 1983

4. Kahan A, Weber S, Amor B, et al: Nifedipine and Raynaud's phenomenon. Ann Intern Med 94:546, 1981

5. Jaffe IA: Nifedipine in digital ulceration in scleroderma. Arthritis Rheum 25:1267, 1982

6. Braunwald E: Mechanism of action of calcium-channel blocking agents. N Engl J Med 307:1618, 1982

7. Mikkelsen E, Anderson K-E, Pedersen OL: The effect of nifedipine on isolated human peripheral vessels. Acta Pharmacol Toxicol 43:291, 1978

8. Mostbeck A, Partsch H, Peschl L: Extracardial effects of nifedipine: Measurements of liver blood flow on animals and humans and of peripheral circulation in the lower limbs: In: First International Nifedipine "Adalat" Symposium: New Therapy of Ischemic Heart Disease. Edited by Hashimoto K, Kimona E, Kobayashi T. Tokyo, University of Tokyo Press, 1970, p 303

9. Schlossman $\mathrm{K}$, Mederwald $\mathrm{H}$, Rosenkranz $\mathrm{H}$ : Investigations on the metabolism and protein binding of nifedipine. Lochnerw, Second International Adalat Symposium, Berlin. Excerpta Medica 33, 1975

10. Antman E, Muller J, Goldberg S, et al: Nifedipine therapy for coronary-artery spasm. N Engl J Med 302:1269, 1980

11. Kahan A, Weber S, Amor B, et al: Nifedipine for Raynaud's phenomenon. Lancet i:131, 1983

12. Smith CD, McKendry RJR: Controlled trial of nifedipine in the treatment of Raynaud's phenomenon. Lancet ii:1299, 1982

13. Rodeheffer RJ, Rommer JA, Wigley F, et al: Controlled doubleblind trial of nifedipine in the treatment of Raynaud's phenomenon. N Engl J Med 308:880, 1983

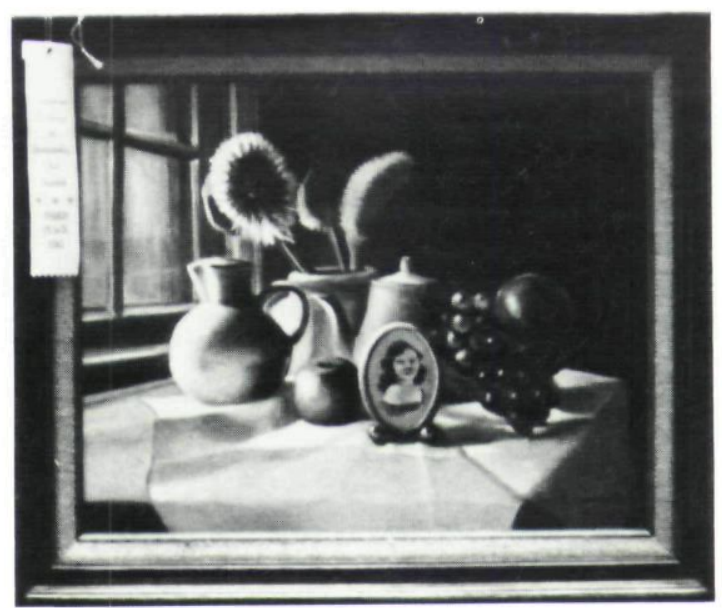

"Still Life, Arlington." Edward M. Shapiro, M.D. Third Place-Oil Painting, 1983. Exhibit of the American Academy of Dermatology, Chicago, Illinois. Photograph courtesy of Hoechst-Roussel Pharmaceuticals, Inc. 
This document is a scanned copy of a printed document. No warranty is given about the accuracy of the copy. Users should refer to the original published version of the material. 